\title{
GRB 101225A - a new class of GRBs?
}

\section{C. Thöne ${ }^{1}$, A. de Ugarte Postigo ${ }^{1,2}$, C. Fryer ${ }^{3}$, K. Page ${ }^{4}$, J. Gorosabel ${ }^{1}$, D. Perley ${ }^{5}$, M. Aloy ${ }^{6}$, C. Kouveliotou ${ }^{7}$ and the Christmas Burst collaboration}

${ }^{1}$ IAA - CSIC, Glorieta de la Astronomía s/n, E - 18008, Granada, Spain email: cthoene@iaa.es

\author{
${ }^{2}$ Dark Cosmology Centre, Juliane Maries Vej 30, DK - 2100 Copenhagen, Denmark \\ ${ }^{3}$ Los Alamos National Laboratory, MS D409, CCS-2, Los Alamos, New Mexico 87545, USA \\ ${ }^{4}$ Dep. of Physics and Astronomy, Univ. of Leicester, University Road, Leicester LE1 7RH, UK \\ ${ }^{5}$ Astronomy Department, UC Berkeley, 601 Campbell Hall, Berkeley, California 94720, USA \\ ${ }^{6}$ Departamento de Astronomia y Astrofisica, Universidad de Valencia, 46100 Burjassot, Spain \\ ${ }^{7}$ Science and Technology Office, ZP12, NASA/MSFC, Huntsville, Alabama 35812, USA
}

\begin{abstract}
The Christmas burst, GRB 101225A, was one of the most controversial bursts in the last few years. Its exceptionally long duration but bright X-ray emission showing a thermal component followed by a strange afterglow with a thermal SED lead to two different interpretations. We present here our model ascribing this strange event to a new type of GRB progenitor consisting of a neutron star and an evolved main-sequence star in a very faint galaxy at redshift 0.33 while Campana et al. (2011) proposed a Galactic origin. New observations at several wavelengths might resolve the question between the two models in the near future.
\end{abstract}

Keywords. gamma rays: bursts, supernovae: individual

\section{Introduction}

Several GRBs in the last years have questioned our original concept of two classes of GRBs first proposed by Kouveliotou et al. (1993) and the subsequent connection with two different types of progenitors. Long-duration GRBs are due to the collapse of a massive star (Woosley \& Bloom 2006), whereas short GRBs come from the merger of two compact objects. While it seems now established that even intrinsically short GRBs (Levesque et al. 2009) or those without SN (Fynbo et al. 2006) can be connected to the death of massive stars, some exceptionally long and soft nearby GRBs have been observed which could be connected to a different kind of progenitor.

The standard fireball model predicts the afterglow emission to come from synchrotron radiation and the SED as well as the temporal evolution are subsequently expected to follow power-laws. A few X-ray afterglows, all connected to nearby long GRBs associated with SNe, showed a thermal component (TC) with a contribution of usually around $20 \%$ (see e.g. Starling et al., this volume). Surprisingly, most nearby GRB-SNe were subluminous (with the exception of GRB 030329) suggesting they might be a different class than the classical "cosmological" GRB. All SNe connected to those nearby GRBs are luminous broad-line Type Ic SNe. Here we present GRB 101225A, called "The Christmas Burst", whose properties largely deviate from those listed above for usual long GRBs. 


\section{Observations of GRB 101225A}

GRB 101225A was discovered by Swift on Dec. 25, 2010 at 18:37:45 UT in an image trigger (Racusin et al. 2010). It had an exceptionally long $\mathrm{T}_{90}$ of at least $2000 \mathrm{~s}$ with indications for emission ongoing for several days (Thöne et al. (2011)) and a very soft spectrum. This puts it at the extreme end of the hardness - duration distribution of Swift GRBs, similar to a few other nearby GRB SNe like GRB 060218 and GRB 100316D.

The X-ray counterpart detected by XRT, the brightest at several thousand seconds, had a very shallow decay in the first 0.2 days before rapidly dropping beyond the detection limit with $\mathrm{t}^{5 / 3}$, inconsistent with predictions from synchrotron radiation. Similar behaviour was observed for GRB 060218 and the recent extremely long GRB 111109A. In first two orbits (up to $\sim 8 \mathrm{ks}$ ) of the X-ray afterglow observation, an additional component to the power-law fit is best modeled with a blackbody $(\mathrm{BB})$ of $\mathrm{T} \sim 1 \mathrm{keV}$ and a radius of $\sim 1 \mathrm{R}_{\odot}$ (assuming a redshift of $\mathrm{z}=0.33$, see below). The $\mathrm{BB}$ component contributes around $20 \%$ to the total flux and shows no temporal evolution.

An optical counterpart was discovered by AlFOSC/NOT 1.54h after the burst (Xu et al. 2010) with nearly constant luminosity over the first 2 days. An extensive UV-opticalIR (UVOIR) follow-up until 2 months after the event revealed a very usual behaviour, both in the light-curve and the SED. Collecting all available UVOIR data, we derive an SED at 7 different epochs from 0.07 to 40 days post burst (Thöne et al. 2011). The SED can not be fit with a single power-law as expected from the fireball model. During the first 10 days, it is well modeled with a simple expanding and cooling BB. The radius of the UVOIR BB increases from 13 to $45 \mathrm{AU}$ while the temperature drops from 43,000 to $5,000 \mathrm{~K}$ from 0.07 to 18 days. The temperature and radius evolution of $\mathrm{X}$-ray and UVOIR $\mathrm{BB}$ are very different suggesting that the emissions come from different processes.

At around 10 days, the simple BB model is no longer valid and an additional, more complicated component becomes evident. At the same time, the light-curve shows a small rise with a maximum at around 30 days before it starts to drop again. This behaviour fits very well to a late, faint supernova associated with the GRB. Spectroscopy at several epochs closely after the GRB and at 41 days did not reveal any absorption or emission lines from the host galaxy nor any broad SN features, therefore, a spectroscopic redshift of the event could not be obtained. We model the SED at 40 days with several SN templates, concluding that a 1998bw-like (Galama et al. 1998) spectrum fits the data best, however stretched in time by a factor of 1.25 and only $1 / 10$ th of the luminosity. From the SED fit, we obtain a photometric redshift of $z=0.33_{-0.04}^{+0.07}$. At $\mathrm{z}=0.33$, the $\mathrm{SN}$ has a luminosity of $-16.7 \mathrm{mag}$, making it the faintest GRB-SN observed.

Preimaging observations of the field from the PAnDAs survey (Richardson et al. 2011) showed a possible object with low significance at $g^{\prime} \sim 27.2$ mag. We reobserved the field 180 days after the burst with OSIRIS/GTC at a total exposure time of $4.1 \mathrm{~h}$. The suggested host candidate from the preimaging is clearly detected, but unresolved, with $g^{\prime}=27.21 \pm 0.27, r^{\prime}=26.90 \pm 0.14$. It shows a blue color, consistent with a star-forming galaxy and lies well above the extrapolation of the light curve (see Fig. 1). Its absolute magnitude of $\mathrm{M}_{g^{\prime}}=-13.7$ mag makes it magnitudes fainter than the host of GRB 060218 .

\section{Our model}

We suggest a new progenitor model to explain all the observed features described in Sect. 2: A merger between an evolved He-star and a neutron star (NS) binary system, which had been suggested as possible GRB progenitor model long ago (Fryer \& Woosley (1998)). When the He-star leaves the main sequence, it expands and incorporates the NS, leading to a common envelope phase during which the He-star ejects most of its envelope 
Table 1. GRBs with SNe and no observed afterglows

\begin{tabular}{lllllllllll}
\hline GRB & $\mathrm{z}$ & $\begin{array}{l}\mathrm{TC}^{2} \\
\mathrm{X}-\mathrm{ray}\end{array}$ & $\begin{array}{l}\mathrm{TC}^{2} \\
\text { optical }\end{array}$ & $\begin{array}{l}\mathrm{T}_{90} \\
(\mathrm{~s})\end{array}$ & $\begin{array}{l}\mathrm{E}_{\text {peak }} \\
(\mathrm{keV})\end{array}$ & $\begin{array}{l}\mathrm{E}_{\text {iso }} \\
(\mathrm{erg})\end{array}$ & $\mathrm{HR}^{3}$ & $\begin{array}{l}\text { Radio? } \\
(\mathrm{mag})\end{array}$ & $\begin{array}{l}\mathrm{SN} \mathrm{M}_{V}{ }^{4} \\
(\mathrm{mag})\end{array}$ & Host $\mathrm{M}_{B}{ }^{5}$ \\
\hline 980425 & 0.0085 & No & $?$ & 23.3 & $55 \pm 21$ & $8.1 \times 10^{47}$ & - & Yes & -19.42 & -17.6 \\
031203 & 0.105 & No & $?$ & 30 & $158 \pm 51$ & $3 \times 10^{49}$ & - & Yes & -20.39 & -21.0 \\
060218 & 0.0331 & Yes & Yes & $\sim 2100$ & 4.9 & $6.2 \times 10^{49}$ & 0.835 & Yes & -18.76 & -15.9 \\
$080109 *$ & 0.0065 & Yes & Yes & $\sim 400$ & low & $2 \times 10^{46}$ & - & Yes & -16.7 & -20.7 \\
$100316 \mathrm{D}$ & 0.059 & Yes & $?$ & $>1300$ & - & $3.1 \times 10^{49}$ & 0.891 & No & -18.62 & -18.8 \\
$101225 \mathrm{~A}$ & 0.33 & Yes & Yes & $>2000$ & $38 \pm 20$ & $>1.4 \times 10^{51}$ & 1.06 & No & -16.9 & -13.7 \\
\hline
\end{tabular}

Notes: ${ }^{1 *}$ No $\gamma$-rays observed, numbers derived from X-rays; ${ }^{2} \mathrm{TC}=$ thermal component; ${ }^{3} \mathrm{HR}=$ hardness ratio defined as $(50-100 \mathrm{keV}) /(25-50 \mathrm{keV}) ;{ }^{4} \mathrm{SN} \mathrm{M}_{V}$ SN peak absolute magnitude in $V$

in form of a thick shell. In our model, this shell must form a broad torus with a narrow opening (funnel) at the rotation axis.

When the two stars merge, an accretion disk and jets are formed leading to a GRBlike event. Only a small part of the jet escapes through the funnel giving rise to the detected $\gamma$-ray emission while most of it interacts with the previously ejected material. Backwards scattered material from the inner boundary of the envelope leads to a hot spot causing the X-ray emission. Most of the jet gets thermalized when interacting with the material in the funnel wall. When the material breaks out of the shell, a hot plume is formed responsible for the UVOIR BB emission, which is finally overtaken by the SN. The model predicts only a small amount of radioactive Ni and hence a faint SN.

We investigated if other GRBs in the past showed a similar behaviour, in particular those without "traditional" afterglow (see Tab. 1). Some of them had a TC in X-rays, although GRBs exist that have a TC in X-rays but show the usual power-law afterglow (e.g. GRB 090618 Page et al. 2011, Cano et al. 2011). Most of these lack early optical data and the onset of the SN is much earlier than for GRB 101225A. The early SEDs of GRB 060218 and XRO 080109, however, both show a TC in the optical during the first days.
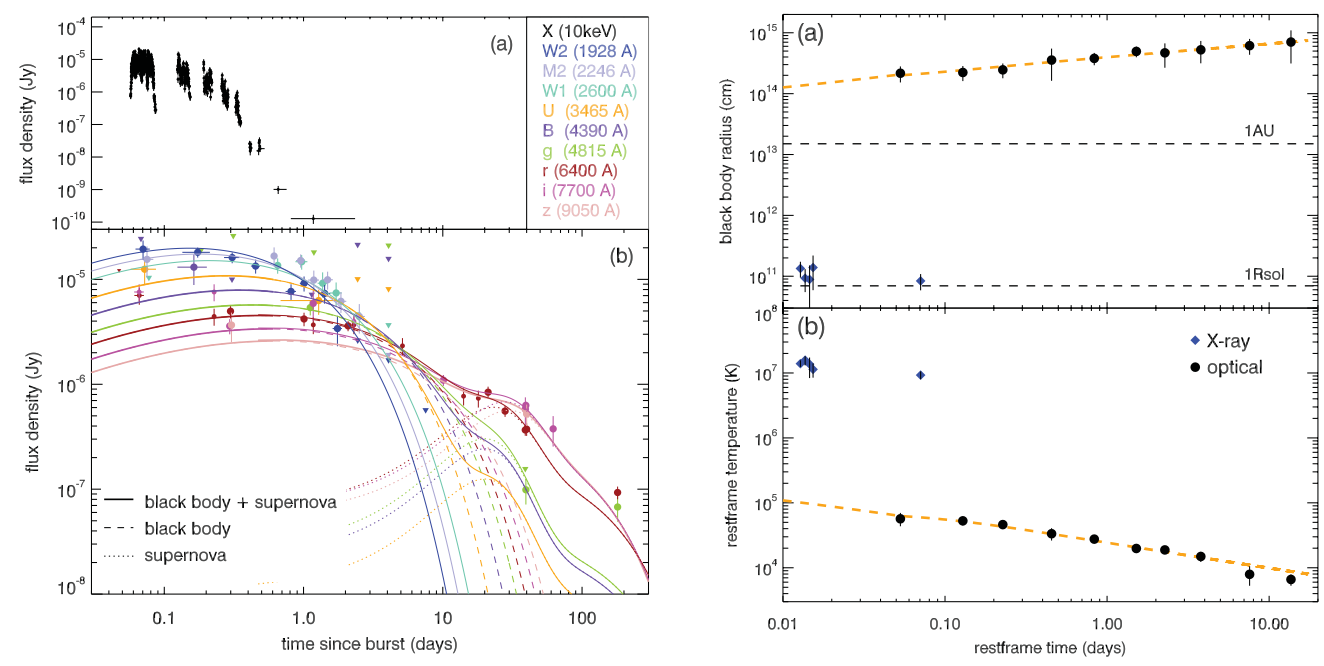

Figure 1. Left: Lightcurve in X-rays, and different UV-optical filters, the contribution of the different emission components described in the text are indicated in the lower panel. Right: Temperature and radius evolution of the X-ray and UVOIR BB emissions together with the predictions from our model (dashed line). 
The UVOIR TC of XRO 080109 seems to be just the continuation of the shock-breakout observed in X-rays Soderberg et al. (2008). GRB 060218 has a UVOIR BB evolution similar to GRB 101225A and the X-ray and UVOIR BB are also inconsistent with a common origin. The X-ray BB radius however is larger, the SN much brighter and also emerges much earlier, while the GRB itself was subluminous. GRB 060218 could come from a similar event with a somewhat different progenitor.

\section{Alternative models and how we can decide between them}

Campana et al. 2011 suggest an entirely different, Galactic, model for this event namely the tidal disruption of a minor body (e.g. a comet) onto an isolated neutron star at $\sim 3 \mathrm{kpc}$. The early emission is explained by contributions from the accretion disk of disrupted comet while the late detection (our host candidate) is ascribed to a proto-planetary disk from which the colliding comet had originated. Another recent event, GRB 111209A, with a duration of $>10 \mathrm{ks}$, showed a remarkably similar X-ray light-curve (Hoversten et al. 2011) together with the same conspicuous "dips" still unexplained for GRB 101225A. The afterglow, however, had a normal power-law behaviour. It could be associated with a galaxy at $\mathrm{z}=0.67$ from emission lines of the host, which itself has so far been undetected. This burst was suggested to come from a tidal disruption (TD) event similar to 100328A (A. Levan priv. comm.).

New late time observations might be able to distinguish between these models. The detection of an extended source, e.g. with HST, would clearly suggest an extragalactic origin, since any disk around a Galactic NS at $3 \mathrm{kpc}$ would be unresolved. A late detection in X-rays would rule out our GRB-like progenitor, the same with a late detection in radio since we do not expect any emission except from the host galaxy at late times. The distinction between the TD model and the GRB model can only definitely be answered if the host can be resolved and the afterglow position is clearly offset from the main emission of the galaxy. Those observations, subject to decisions by different time allocation committees, will hopefully reveal the nature of this strange event in the near future.

Acknowledgements: CCT acknowledges support from "Estallidos" under program number AYA2010-21887-C04-01 of the Spanish MEC and by FEDER and generous support from the IAU to attend this conference.

\section{References}

Campana, S. et al., 2011, Nature, 480, 69

Kouveliotou, C. et al. 1993, ApJ, 413, L101

Woosley, S. E. \& Bloom, J. S. 2006, ARAA, 44, 507

Levesque, E. M., et al. 2010, MNRAS, 401, 963

Fynbo, J. P. U. et al. 2006, Nature, 444, 1047

Racusin, J. L. et al. 2010, GCN Circ. 11493

Thöne, C. C. et al. 2011, Nature, 470, 72

Xu, D., Ilyin, I., \& Fynbo, J., 2010, GCN Circ., 11495

Galama, T. J. et al. 1998, Nature, 395, 670

Richardson, J. C. et al. 2011, ApJ, 732, 76

Fryer, C. L. \& Woosley, S. E. 1998, ApJ, 502, L9

Campana, S. et al. 2006, Nature, 442, 1008

Page, K. L. et al. 2011, MNRAS, 416, 2078

Cano, Z. et al. 2011, MNRAS, 413, 669

Soderberg, A. et al. 2008, Nature, 453, 469

Hoversten, E. A. et al. 2011, GCN Circ. 12641 